\title{
Towards using UAV for improving 5 G cellular communication systems
}

\author{
Mohamed El Sayed Kotb ${ }^{1}$, Wagdy R. Anis ${ }^{2}$, Ahmed A. Abd-Elhafez \\ ${ }^{1}$ Student in Faculty of Engineering-Ain-Shams University (ASU), Egypt \\ ${ }^{2}$ Professor in Faculty of Engineering Ain-Shams University (ASU), Egypt \\ ${ }^{3}$ Communications Department, Military Technical Collage (MTC), Egypt
}

\section{Article Info}

Article history:

Received Feb 10, 2021

Revised Jun 5, 2021

Accepted Jun 11, 2021

\section{Keywords:}

Cellular network

Ground control station

Payload

Terrestrial communications

Unmanned aerial vehicles

\begin{abstract}
Unmanned aerial vehicles (UAVs) have sparked a lot of interest in the wireless networking community as an emerging subject in aerial robotics. The UAV environment can be used to improve UAV communications in various ways. These smart devices cater for a broad variety of wireless technologies and applications because of UAV's inherent features related to versatile mobility in 3D space, autonomous operations as well as intelligent positioning. This study will investigate the convergence synergies between 5G/B5G mobile systems and UAV technologies, with the UAV being integrated into current cellular networks as a modern aerial user equipment (UE). In this integration, UAVs play the function of cellular flying customers, and are hence referred to as cellularly linked UAVs (a.k.a. UAVUE, drone-UE, 5G-connected drone, or aerial user). The major goal of this research is to provide a thorough analysis of the integration task, as well as major technical breakthroughs from 5G/B5G and current work in prototyping architecture and field trials that support cell-based UAVs. This study examines recent 3GPP standards advances as well as socio-economic challenges that must be addressed before this promising technology can be properly implemented. There are already some accessible issues clearing the way for potential study opportunities.
\end{abstract}

This is an open access article under the $\underline{C C B Y-S A}$ license.

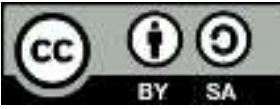

\section{Corresponding Author:}

Mohamed El Sayed Kotb El Sayed

Department of Electronic and communication Engineering

Faculty of Engineering, Ain-Shams University (ASU), Egypt

Email: mohammed.kotp2@gmail.com

\section{INTRODUCTION}

Unmanned aerial vehicles, or UAVs, are typically operated and handled by aircraft that do not have a human pilot on board or by embedded autonomous computer programmes. Drones are a common term for unmanned aerial vehicles (UAVs). It is a cutting-edge concept derived from aerospace robotics that has enormous potential for novel applications and economic opportunities in a variety of industries [1]-[3]. The global UAV market was assessed to be US\$20.68 billion in 2017 and is anticipated to reach US\$59.82 billion by 2026 [4], with a cumulative annual growth rate (CAGR) of 14.20 percent during the projected era.

Three-dimensional, autonomous, and modular UAVs have been developed to provide appealing solutions for a wide range of applications, including civil and public safety, industrial IoT networks, security and defence industries, cyber physical structures, atmospheric and environmental observation, and so on [5]-[7]. UAVs have been able to exhibit substantial use across a variety of civilian and industrial applications in many domains using new technologies such as artificial intelligence (AI), the internet of things (IoT), and augmented reality/virtual reality (AR/VR). Because UAVs are adaptive altitude-supporting 
flying platforms, new use cases for each of these applications are emerging, such as safe and accurate wireless guiding and control coordination, as well as effective knowledge transmission to the ground control system [8]-[10].

In order for this next development to take place, two lines of investigation are needed. First, how a wireless networking network that is designed and integrated to meet the specific needs of drones while enabling accessibility and easy operation for numerous applications is applied [11]-[13]. Another important factor to consider is the extent to which scientific and technological problems arise from this merger. To this end, we will focus on demonstrating distinctive characteristics and accompanying challenges [14]-[16].

\subsection{Requirement for UAV communication}

UAV requirements can be categorised into two groups from the standpoint of contact [17]:

- Communication control and non-payload (CNPC): This refers to flight operations' time-critical control and safety controls. To maintain a stable and effective UAV service, CNPC requires navigational updates, waypoint updates, and ATC updates. CNPC typically needs very safe and efficient connectivity with low data rate requirements (few hundred $\mathrm{Kb} / \mathrm{s}$ ). The $\mathrm{CNPC}$ reliability criterion is less than a packet error rate of 10-3 (PER).

- Payload communication: This refers to any knowledge dissemination procedures between the UAV and land stations that are part of a UAV mission. For example, in a monitoring procedure, a UAV must transmit real-time video to a base station/remote pilot via payload communication. Payload communication necessitates the use of a media capable of handling high data rates (often higher in full HD video transmission or wireless backhauling) [18].

\subsection{Integration opportunities with cellular network}

UAV penetration into the mobile network is one of three broad paradigms [19], [20]:

- UAV-assisted cellular communications: UAVs are intended to serve as flying base points, relays, or anchor locations that may intelligently relocate themselves to improve the visible quality of experience (QOE), spectrum dependability, and coverage gains of present wireless terrestrial networking systems [21], [22]. This architecture can be seen in Figure 1 (a). Because of its dynamic mobility and repositioning, the adoption of UAVs provides a wide variety of benefits to the current terrestrial contact scheme. On request, the UAV's base station (fly-in base station or relays) could be provided, which is an attractive option for disaster management. To address the needs of consumers in hotspot areas, optimal 3D positioning and synchronisation of flying base stations will expand the reach and data rate of current wireless networks. These advantages undoubtedly satisfy complex, dynamic and growing data requirements for cellular $5 \mathrm{G} / \mathrm{B} 5 \mathrm{G}$ networks.

- Communications with cellular assisted UAVs: This is also known as mobile unmanned aerial vehicles (UAVs). Flying UAVs, as shown in Figure 1 (b), are current aerial user systems (UEs) of terrestrial UEs that connect to the network networks of the sky's cells. In recent years, due to its success in establishing secure wireless communications with land cellular stations, this approach has received a lot of attention [23].

- UAV-UAV communication-UAV communication: In this model, a community of UAVs interacts with the ground user in a trustworthy manner in order to encourage autonomous flight, fleet coordination, and collision avoidance. Figure 1 (c), depicts the architecture. The authors looked at secure and direct communications between UAVs that use the same radio spectrum and connect cellular soil users [24].

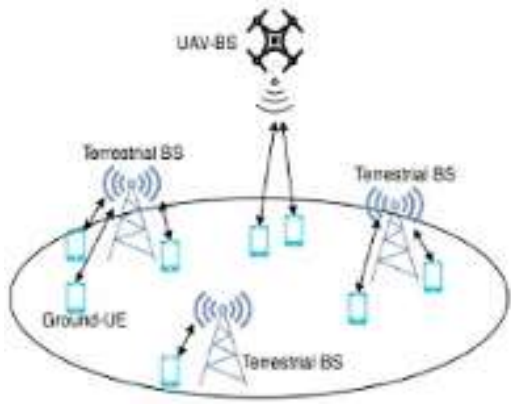

(a)

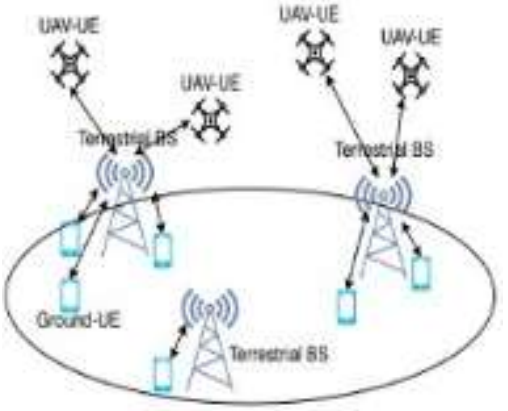

(b)

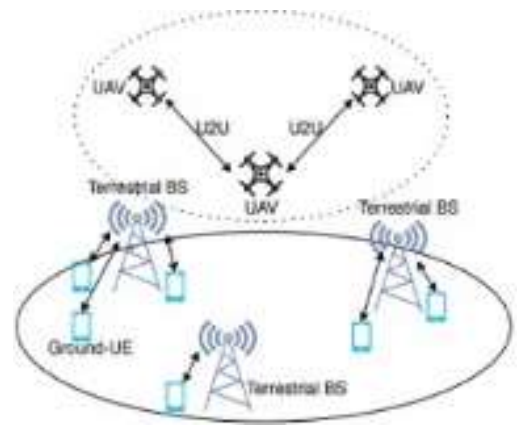

(c)

Figure 1. UAV-cellular network integration opportunities, (a) UAV-assisted cellular communication, (b) Cellular-assisted UAV communication, (c) UAV-UAV communication 


\section{RESEARCHS AND TUTORIALS RELATED}

Work is on the increase to explore the interplay between UAVs and cellular networks. In recent years, new ideas have been proposed to address the problems of science, technological, socio-economic and security issues. The literature includes many polls, presentations and guides that offer a coherent understanding of this study field. These works not only allow the research group to monitor continuous research, but also to bring together the knowledge that practitioners and researchers in the community require.

The majority of the research and tutorials are primarily focused on: i) UAV integration opportunities with 5G/B5go cellular networks from a UAV-assisted cellular communication perspective, ii) new developments, future trends, and challenges for UAV cellular communication, or iii) detailed analysis and performance study with regard to specific communication problems, such as the canal. Our dissertation, on the other hand, is focused on the cellular UAV communications concept. We want to outline our existing research and tutorials, as well as work on cellular UAVs, to emphasise the importance and novelty of our new researchs in comparison to previous research.

The majority of current researchs are focused on and discussed in detail on UAV-assisted cellular contact. There is a small number of researchs focusing on the cellular UAV model, However, the current works are dispersed and do not provide a comprehensive understanding of the paradigm. In other words, only a few specific aspects of cellular UAV connectivity have been thoroughly investigated thus far, such as UAV-ground channel modelling or optimization trajectory or MIMO. These works do not offer a comprehensive analysis, with review highlights of all sorts devoted to mobile UAVs, but rather a unique subject in detail. Therefore, a cohesive work is still lacking and provides an overview of all types of research progress.

This research aims to fill this void and focuses exclusively on cellular UAVs. The research includes analysis on state-of-the-art technologies, synergistic convergence problems for UAVs as aerial users in 5G/ B5G mobile networks, the underlying network architectures, 5G improvements to physical layers, field testing, simulation and test bed creation. Network virtualization functions interaction (NFV) In the architectural context of 5G/B5G progress, cloud infrastructure technology for mobile linked UAVs also provides the cloudification and software of network resources, promoting them.

\section{RESULTS AND DISCUSSION}

It is nearly impossible to simulate all that goes on in the real world with real-world UAVs, including the unique challenges and advantages of those aircrafts linked by cellular networks. There is a deficiency of academic literature. Various ongoing efforts are undertaken by academics from both the business and academia who are focusing on more practical prototypes that will likely expand the capabilities of these ongoing technologies [25]. The significance of the most often differs depending on (on) the purpose for which the concept or objective that the prototypes are designed and (as compared to the other concepts/objectives that are realized), and function implementation ( $\mathrm{s}$ are, in turn, often reliant on the main objective. By way of example, one, a research project may concentrate fully investigate 5G/5G network operations and efficient use of unmanned aerial vehicles (UAVs) 5 and the. Another prototype can place priority on having an efficient, predictable connectivity with good quality of service (QoS) guarantees.

Additionally, each prototype is able to implement separate hardware and software flight stacks to reach the same end target. Although it is up to the owners to choose the type of platforms they use, Open source or proprietary hardware and software are both possible. Thus, current initiatives focus on goals have a strong details and purpose to the product that make them more valuable or interesting to customers, contributing meaning and traits to the design of the product being designed. Before there are official standards available, we must pursue these prototyping efforts without regard to the need for compatibility or the possibility of conflicts.

But we're still lacking an optimal representation of cellular-connected drone prototype. Although these particular features of the perfect prototype would depend on project requirements, the goal is important: the product must have a set of critical features that are not too complex. So far, our study has done exactly nothing but presume an optimal and list all-inclusive prototype, and investigate the factors that go into it. a prototype is referenced to reveal the features that users may have and make a comparison with previous products to outline the features to which end users would like to have in a given context a completely nonexhaustive list of these features attempts to give you an analogy for this product is meant to serve as a utilitarian purpose, not as an exhaustive specification. Examples of new to existing use cases, particularly new features, can be discovered through the evolution of evolving applications for cellular-connected UAVs.

We are specifically looking at what exists in the architecture and development of current and potential uses of UAVs with respect to $\mathrm{LTE} / 4 \mathrm{G} / 5 \mathrm{G} / \mathrm{B}$ over cellular network networks here. The queries are 
outlined in the following ways. And about 90 percent open-source $4 \mathrm{G}$ connected devices were tested, free and lightweight UAV is defined, bringing forth a new, light weight, stable, and lightweight class of cellular connected vehicle. To deliver a fully autonomous and fail-safe flying, this design and development integrates open-source hardware and open-software hardware/open-software building blocks. This contains a comprehensive explanation of flight controls, radio antennas, and $4 \mathrm{G}$ cellular modem hardware and software options, as well as on cellular modems that use $4 \mathrm{G}$ and expand this schematic, the hardware and software components employed in the prototype development are depicted in Figure 2. An intricately designed and highly complex avionics schematic is seen in Figure 3, while the equipment diagrams and detail schematics are tabulated in Table 1. The prototype is examined to make sure it has adequate flight times, stays level, knows which direction the wind is blowing, performs in the air autonomously, and gives real-time video images. The significant developments that have taken place in this work are listed below:

4. Commercial off the shelf (COTS) hardware and applications.

5. For the record, the longest time, the UAV has been aloft in the air for over an hour.

6. Working in tandem with programming, this document shows users the straightforward, succinct and precise path to concept and initial design, which lays out the total course of whole project creation from start to finish. There is an interactive online manual (wiki) and supplementary material to this page.

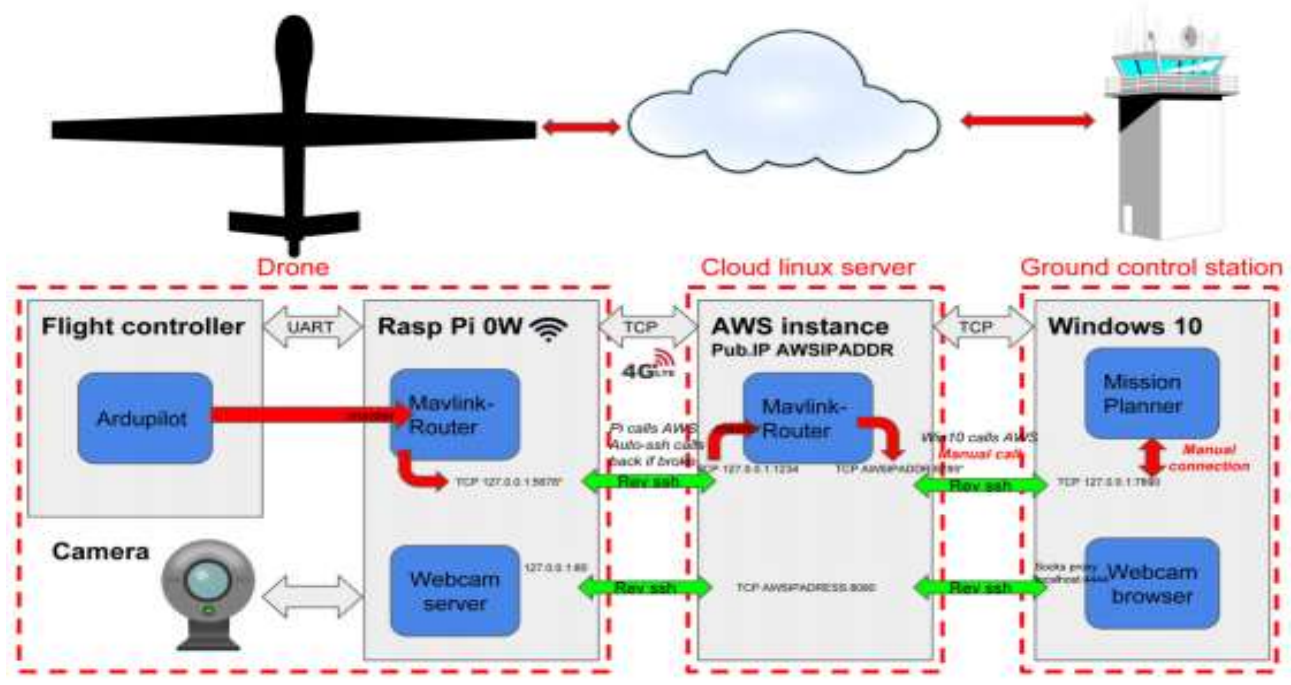

Figure 2. Prototype design and configurations

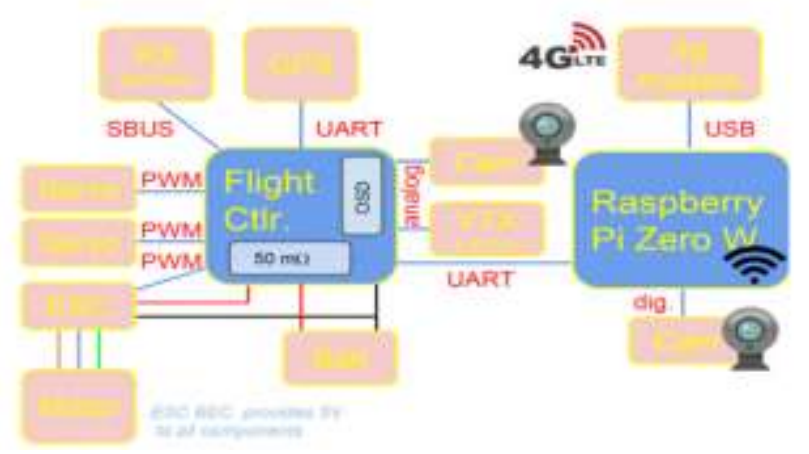

Figure 3. Schematic of the avionics components

Cellular-connected UAVs typically have a variety of Sensors that record and disseminate data are common on cellular-connected UAVs which changes their locations continuously according to these recorded data as shown in Figure 4 between cells and this movement had been simulated as shown in Figures 5 and 6 (see in appendix) to clarify this research attitude and explain the mentioned flying platform. This increases the likelihood of vulnerability exposure. This flying platform is subject to cyber-physical 
attacks aiming at obtaining, inspecting, and manipulating information about UAV payloads by reprogramming it to perform undesirable acts. This flying platform is vulnerable to cyber-physical attacks aimed at stealing, inspecting, and abusing UAV payload details by reprogramming it to do undesirable actions. Existing safety information measures are not suitable for mobile UAVs since they do not take account of potential risks to several on-board sensors and UAV actuators. An intruder can exploit the contact and control mechanism of UAV and therefore make bringing it back online very challenging. New security methodologies must also be established in order to prevent above-mentioned intrusions and hacking procedures.

Table 1. Shows the avionics components that were used

\begin{tabular}{cc}
\hline Component & Model \\
\hline Flight Controller & Omnibus F4 Pro \\
GPS & BN-220 \\
Radio Rx & TBS Nano \\
Camera \& Video Tx & TX05 \\
Computer & Raspberry Pi Zero W \\
4G Modem & Verizon USB730L \\
4G Antenna & TS9 \\
\hline
\end{tabular}

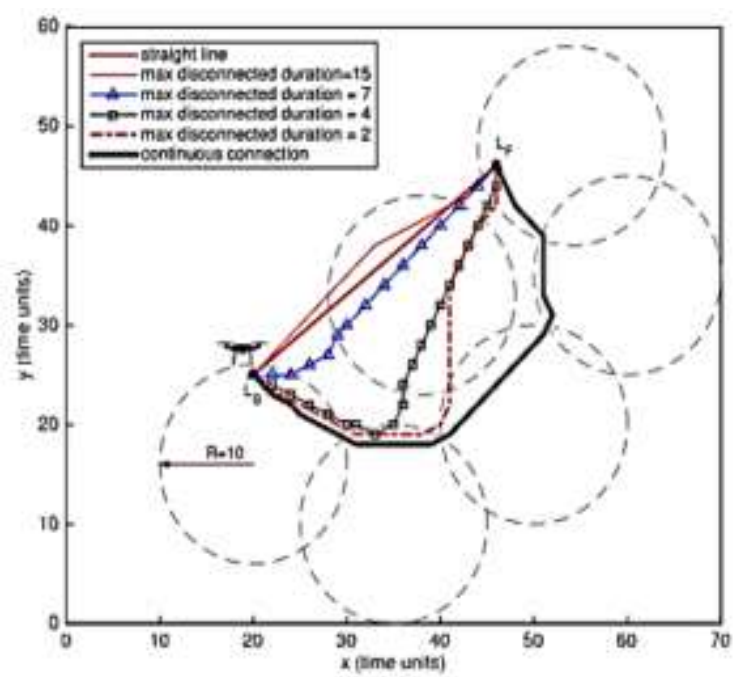

(a)

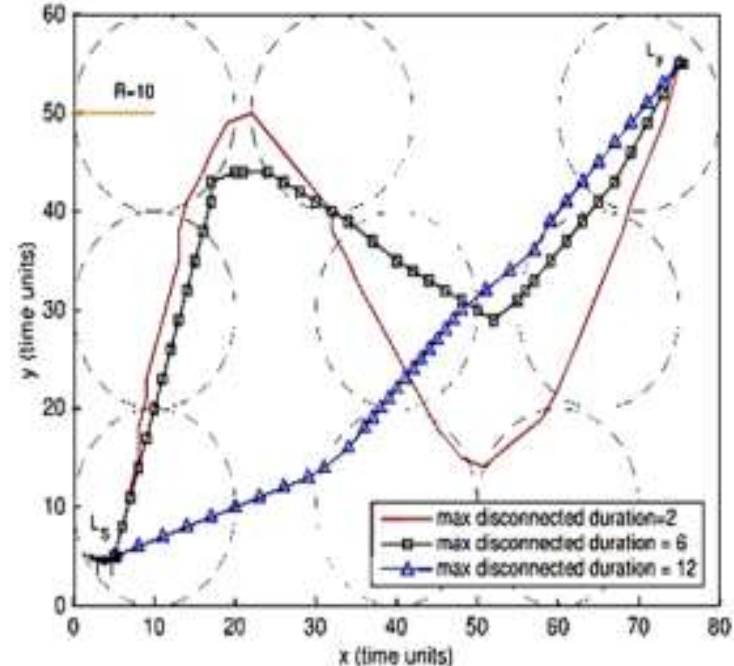

(b)

Figure 4. UAV direction with a discontinuity threshold with two separate cellular layouts, (a) UAV trajectory with first cell layout, (b) UAV trajectory with second cell layout

\section{CONCLUSION}

In this paper, we have conducted an extensive analysis on the UAV connectivity model (cellular linked UAV) where UAV is embedded as a modern aerial UE in established 5G/B5G cells. Firstly, the comprehensive taxonomy of the different technology fields of new applications as well as the technological problems of UAV incorporation with cellular networks. We then concentrate on promising network designs and physical layer enhancements for 5G/B5G networks in view of cellular linked UAVs hardware and device design challenges. The core advanced $5 \mathrm{G}$ solutions have been elaborated such that UAV connectivity across mobile spectrum is integrated and facilitated seamlessly. To assess the design efficiency benefit and Research real implementation challenges, we have highlighted endeavours to build working prototypes, as well as field tests and modelling. Progress in 3GPP standardization operations, national and foreign legislation and socioeconomic hurdles issues will need to be considered before the latest technology can be adopted successfully. We hope that this study would be a valuable and inspiring aid for researchers in mobile connected UAVs to open a holistic picture and harness their maximum potential. 


\section{APPENDIX}

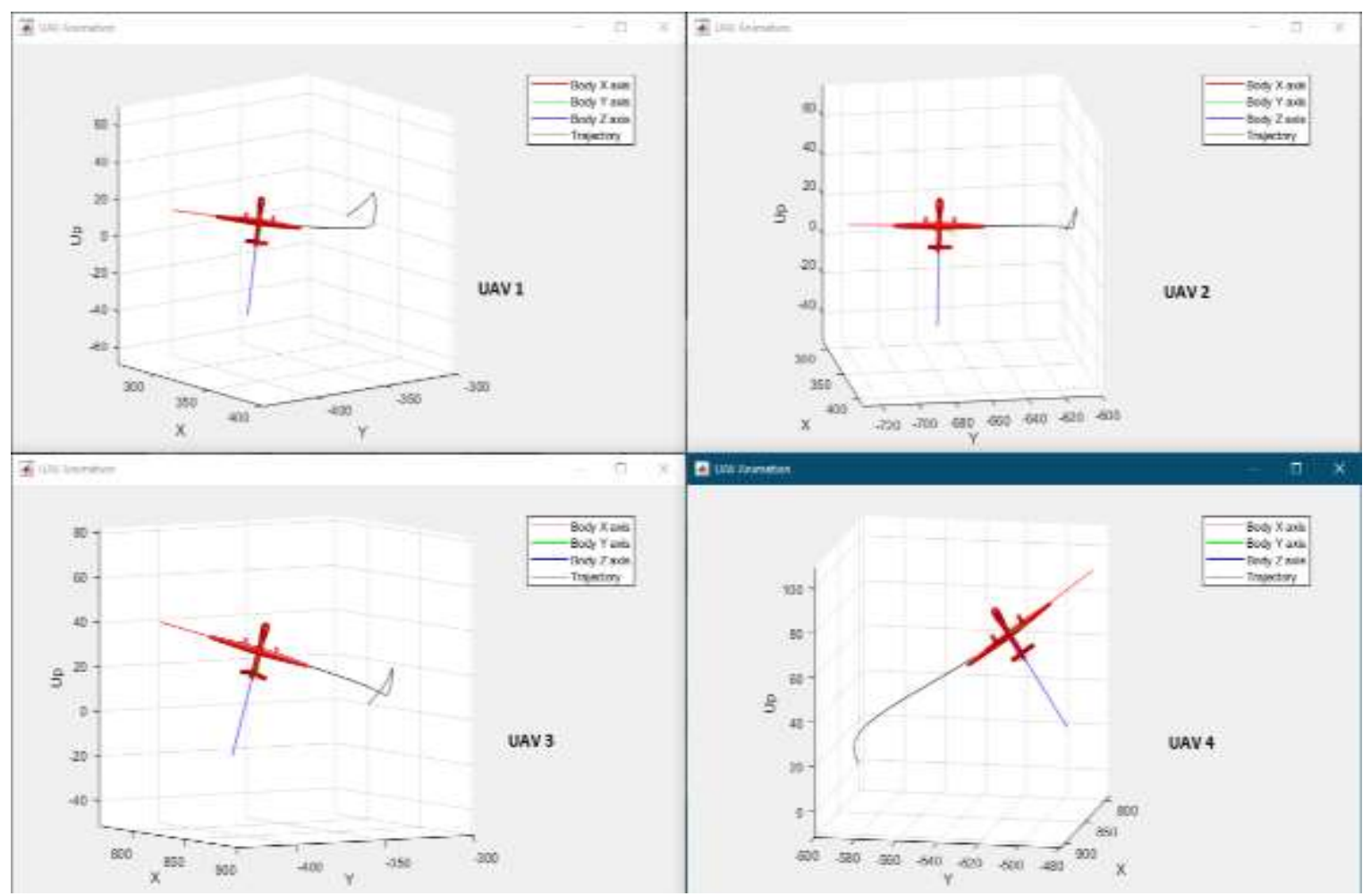

Figure 5. MATLAB animation

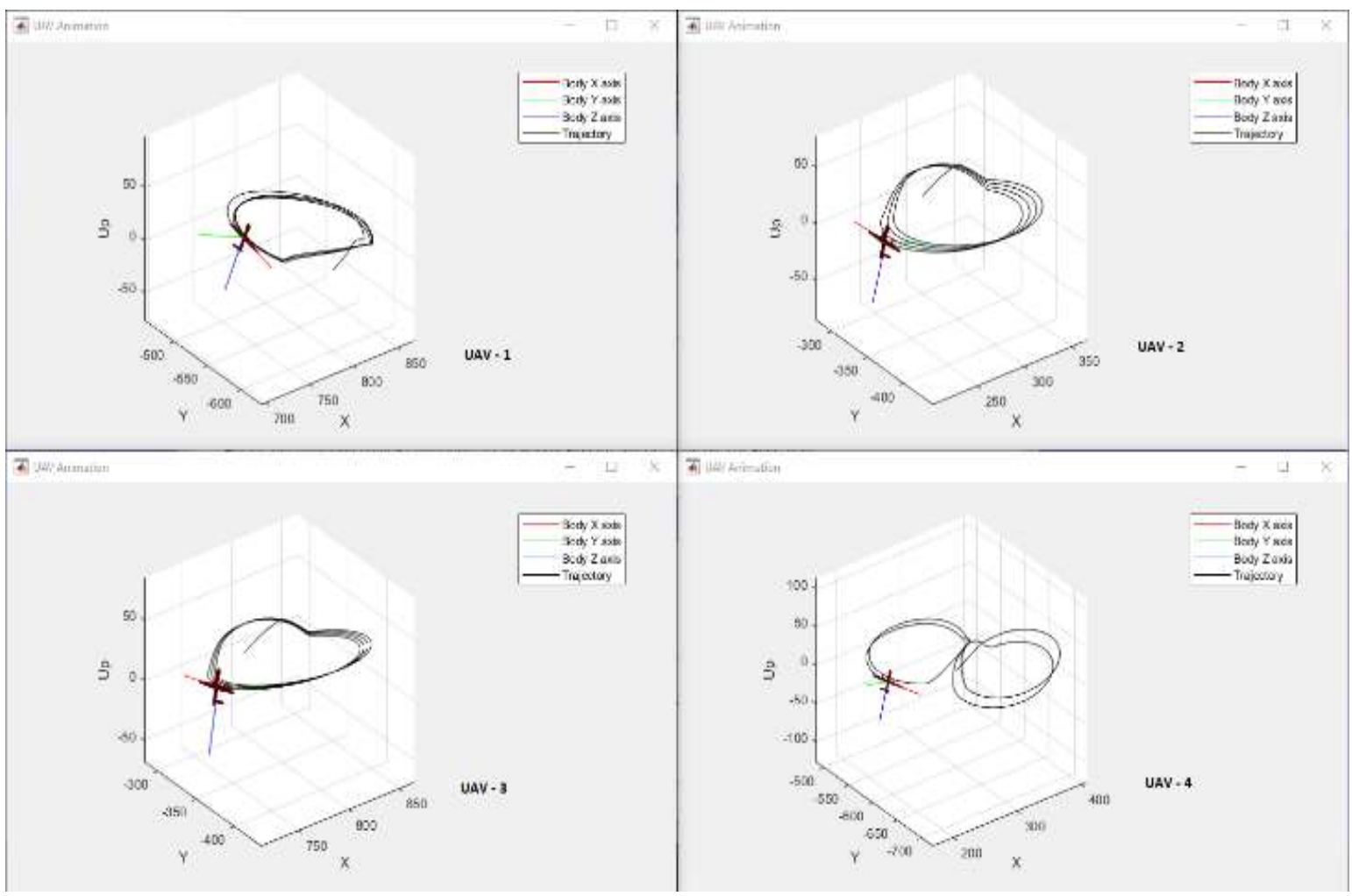

Figure 6. End of simulation 


\section{REFERENCES}

[1] K. P. Valavanis, G. J. Vachtsevanos, "Handbook of unmanned aerial vehicles," Springer, vol. 1, no. 5/6, pp. 43-47, 2015.

[2] R. W. Beard, T. W. McLain, "Small unmanned aircraft: Theory and practice," Princeton university press, 2012.

[3] M. Asadpour, B. Van den Bergh, D. Giustiniano, K. A. Hummel, S. Pollin and B. Plattner, "Micro aerial vehicle networks: An experimental analysis of challenges and opportunities," IEEE Communications Magazine, vol. 52, no. 7, pp. 141-149, 2014, doi: 10.1109/MCOM.2014.6852096.

[4] L. Godage, "Global Unmanned Aerial Vehicle Market (UAV) Industry Analysis and Forecast (2018-2026)," Montana Ledger, 2019.

[5] S. Hayat, E. Yanmaz, R. Muzaffar, "Survey on unmanned aerial vehicle networks for civil applications: A communications viewpoint," IEEE Communications Researchs and Tutorials, vol. 18, no. 4, pp. 2624-2661, 2016, doi: 10.1109/COMST.2016.2560343.

[6] R. Shakeri et al., "Design challenges of multi-UAV systems in cyber-physical applications: A comprehensive research and future directions," IEEE Communications Researchs and Tutorials, vol. 21, no. 4, pp. 3340-3385, 2019, doi: 10.1109/COMST.2019.2924143.

[7] H. Wang, H. Zhao, J. Zhang, D. Ma, J. Li and J. Wei, "Research on unmanned aerial vehicle networks: A cyber physical system perspective," IEEE Communications Researchs and Tutorials, vol. 2, no. 5, pp. 142-168, 2019.

[8] M. Mozaffari, W. Saad, M. Bennis, Y.-H. Nam and M. Debbah, "A tutorial on uavs for wireless networks: Applications, challenges, and open problems," IEEE communications researchs and tutorials, vol. 21, no. 3, pp. 2334-2360, 2019, doi: 10.1109/COMST.2019.2902862.

[9] Y. Zeng, R. Zhang, T. J. Lim, "Wireless communications with unmanned aerial vehicles: Opportunities and challenges," IEEE Communications Magazine, vol. 54, no. 5, pp. 36-42, 2016, doi: 10.1109/MCOM.2016.7470933.

[10] A. Fotouhi et al., "Research on uav cellular communications: Practical aspects, standardization advancements, regulation, and security challenges," IEEE Communications Researchs and Tutorials, vol. 21, no. 4, pp. 3417-3442, 2019, doi: 10.1109/COMST.2019.2906228.

[11] P. Marsch et al., "5G radio access network architecture: Design guidelines and key considerations," IEEE Communications Magazine, vol. 54, no. 11, pp. 24-32, 2016, doi: 10.1109/MCOM.2016.1600147CM.

[12] P. Marsch, I. Da Silva, Ö. Bulakci, M. Tesanovic, S. E. El Ayoubi and M. Säily, "Emerging network architecture and functional design considerations for 5G radio access," Transactions on Emerging Telecommunications Technologies, vol. 27, no. 9, pp. 1168-1177, 2016, doi: 10.1002/ett.3073.

[13] S. D. Muruganathan, X. Lin, H.L. Maattanen, Z. Zou, W. A. Hapsari and S. Yasukawa, "An overview of 3GPP release-15 study on enhanced LTE support for connected drones," arXiv preprint arXiv:1805.00826, 2018.

[14] B. Li, Z. Fei and Y. Zhang, "UAV communications for 5G and beyond: Recent advances and future trends," IEEE Internet of Things Journal, vol. 6, no. 2, pp. 2241-2263, 2018, doi: 10.1109/JIOT.2018.2887086.

[15] I. Bor-Yaliniz, M. Salem, G. Senerath and H. Yanikomeroglu, "Is 5G ready for drones: A look into contemporary and prospective wireless networks from a standardization perspective," IEEE Wireless Communications, vol. 26, no. 1, pp. 18-27, 2019, doi: 10.1109/MWC.2018.1800229.

[16] S. A. R. Naqvi, S. A. Hassan, H. Pervaiz and Q. Ni, "Drone-aided communication as a key enabler for 5G and resilient public safety networks," IEEE Communications Magazine, vol. 56, no. 1, pp. 36-42, 2018, doi: 10.1109/MCOM.2017.1700451.

[17] R. J. Kerczewski, J. D. Wilson and W. D. Bishop, "Frequency spectrum for integration of unmanned aircraft," IEEE/AIAA 32nd Digital Avionics Systems Conference (DASC), 2013, pp. 6D5-1, doi: 10.1109/MAES.2010.5546294.

[18] 3GPP TR 36.777, "Technical specification group radio access network: study on enhanced LTE support for aerial vehicles," vol. 15, 2017.

[19] Y. Zeng, Q. Wu and R. Zhang, "Accessing from the sky: A tutorial on UAV communications for 5G and beyond," Proceedings of the IEEE, vol. 107, no. 12, 2019, pp. 2327-2375, doi: 10.1109/JPROC.2019.2952892.

[20] E. Vinogradov, H. Sallouha, S. De Bast, M. M. Azari and S. Pollin, "Tutorial on UAV: A blue sky view on wireless communication," arXiv preprint arXiv:1901.02306, 2019, doi: 10.13052/jmm1550-4646.1443.

[21] A. Chakraborty, E. Chai, K. Sundaresan, A. Khojastepour and S. Rangarajan, "SkyRAN: a self-organizing LTE RAN in the sky," Proceedings of the 14th International Conference on emerging Networking EXperiments and Technologies, ACM, 2018, pp. 280-292.

[22] K. Sundaresan, E. Chai, A. Chakraborty and S. Rangarajan, "SkyLiTE: End-to-End Design of Low-Altitude UAV Networks for Providing LTE Connectivity," arXiv preprint arXiv:1802.06042, 2018.

[23] Y. Zeng, J. Lyu and R. Zhang, "Cellular-connected UAV: Potential, challenges, and promising technologies," IEEE Wireless Communications, vol. 26, no. 1, pp. 120-127, 2018, doi: 10.1109/MWC.2018.1800023.

[24] M. M. Azari, G. Geraci, A. Garcia-Rodriguez and S. Pollin, "Cellular UAV-to-UAV communications," IEEE 30th Annual International Symposium on Personal, Indoor and Mobile Radio Communications (PIMRC), 2019, pp. 1-7.

[25] N. S. Team, "NetSim interfacing with MATLAB's Simulink for UAV Simulation : NetSim Support Portal," 2021. [Online]. Available: https://support.tetcos.com/support/solutions/articles/14000121929-netsim-matlab-interfacingfor-uav-drone-flying-adhoc-network-simulations, 2021. 


\section{BIOGRAPHIES OF AUTHORS}

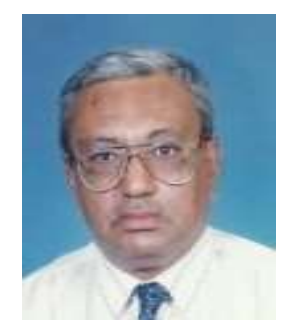

Prof. Dr. Wagdy R. Anis, he received his B.Sc. from the Electrical Engineering Department, Ain shams university, Cairo, Egypt. M.Sc. from Ain shams university in 1977. Ph.D. in 1985 Currently Emeritus Professor at Electronics Engineering and Electrical Communications. Email: wagdianis51@eng.asu.edu.eg

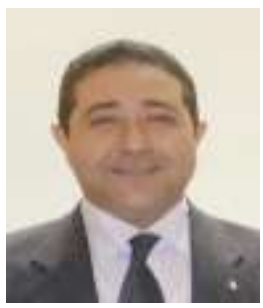

Ass. Prof. Dr. Ahmed A. Abdel-Hafez, he received his B.Sc. and M.Sc. from the Communication Engineering Department, Military Technical College, Cairo, Egypt in 1990, 1997 respectively. Ph.D. from Ottawa University in 2003. Currently Head of cryptography research center since 2012, Egyptian Armed Forces. His research interests including Applied cryptograph, and Information Security. Email: aabdelhafez@gmail.com

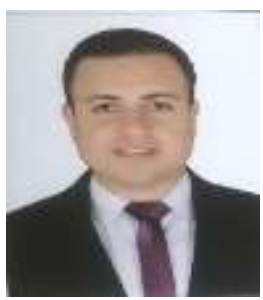

Mohamed El Sayed Kotb El Sayed, he received his B.Sc. from the Communication Engineering Department, faculty of engineering, zagazig university, zagazig, Egypt in 2010, master student in Ain shams university communication and electronics Department. Currently, member of cryptography research center since 2015, Egyptian Armed Forces. Email: mohammed.kotp2@gmail.com 